\title{
Preimplantation Genetic Diagnosis in Isolated Sulfite Oxidase Deficiency
}

\author{
Mustafa A. Salih, Thomas M. Bosley, Ibrahim A. Alorainy, Mohamed A. Sabry, \\ Mohamed S. Rashed, Eiman A. Al-Yamani, Siham El-Akoum, Sarar H. Mohamed, \\ Khaled K. Abu-Amero, Ali M. Hellani
}

Can J Neurol Sci. 2013; 40: 109-112

Isolated sulfite oxidase deficiency (ISOD; OMIM 272300) is an autosomal recessive disorder characterized clinically by neonatal onset of intractable seizures and profound neurodevelopmental retardation with neuropathologic and neuroradiologic features that make this disorder part of the differential diagnosis of hypoxic ischemic encephalopathy ${ }^{1}$. Patients rapidly develop microcephaly, and death often intervenes at an early age due to consequences of severe brain damage.

Sulfite oxidase is a mitochondrial molybdohemoprotein that catalyzes the oxidation of inorganic sulfite to sulfate, the final step in the degradation of sulfur-containing amino acids and environmental sulfites. The gene responsible for sulfite oxidase (SUOX; OMIM 606887) maps to chromosome 12q13.2 and can now be sequenced either pre-partum or post-partum in patients with suspected ISOD. A related autosomal recessive disorder, molybdenum cofactor deficiency (MOCOD; MIM \#252150), also inhibits the action of sulfite oxidase and causes similar clinical, radiologic, and biochemical problems ${ }^{1}$. MOCOD disturbs the metabolism of xanthine to uric acid in addition and has a somewhat different biochemical signature (Figure 1).

We describe a consanguineous family followed for eight years with multiple children affected by ISOD due to a novel SUOX mutation. Preimplantation genetic diagnosis (PGD) and in vitro fertilization resulted in a normal child.

\section{Clinical Report}

Figure 2 illustrates the pedigree of this consanguineous Sudanese family followed for eight years who had three children with clinically and biochemically defined ISOD, two of whom were genetically confirmed. The first pregnancy was a female child (Individual 9) who was aborted therapeutically at five months of gestation because of anencephaly. Their second child was a female (Individual 10) born prematurely at 24 weeks gestation who developed an intraventricular hemorrhage and died at two weeks postpartum without a definitive diagnosis.

Their third child was a girl (Individual 11) born at full-term who developed seizures on the second day of life. She became microcephalic with spastic upper and lower extremities and exaggerated deep tendon reflexes. Brain computed tomography (CT) at the age of 11 months showed loss of white matter and white matter cysts mainly in the corona radiata and centrum semiovale, enlarged cerebral sulci and lateral ventricles, faint calcification in the thalami, and massive dilatation of the ventricular system and cisterna magna. Bilateral lens

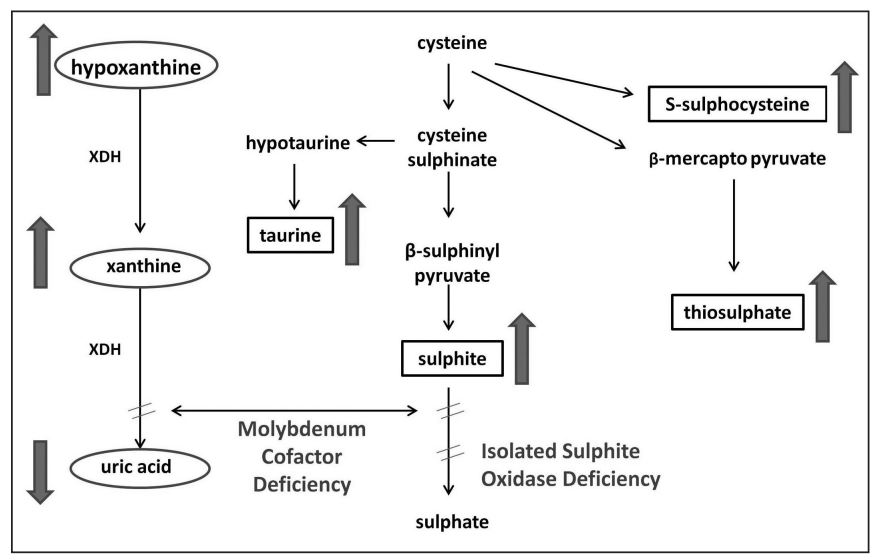

Figure 1: ISOD and MOCOD biochemistry. Diagram outlining biochemical compounds related to sulfate metabolism. Isolated sulfite oxidase deficiency (ISOD) and Molybdenum Cofactor Deficiency (MOCOD) both interfere with the metabolism of sulfite to sulfate by sulfite oxidase. This results in elevated serum and urinary levels of sulfite, S-sulfocysteine, thiosulfate, and taurine as indicated. Molybdenum Cofactor Deficiency also interferes with the metabolism of xanthine to uric acid through xanthine dehydrogenase, resulting in increased levels of xanthine and hypoxanthine and decreased levels of uric acid in this disorder.

subluxation compatible with ISOD was noted, and urinary Ssulphocysteine level was documented to be $326 \mu \mathrm{mol} / \mathrm{mmol}$ of creatinine (control <10). She died at age 14 months of respiratory complications.

Their fourth child (Individual 12) was healthy until the third day of life, when he developed generalized tonic-clonic seizures, decreased activity, and poor feeding with obviously increased muscle tone. Brain CT at the age of four days showed generalized brain swelling involving supratentorial structures

\footnotetext{
From the Pediatrics (Neurology) (MuAS), Ophthalmology (TMB, KKA), Radiology Departments (IAA,), College of Medicine, King Saud University; Department of Genetics (MoAS, MSR, EAA) King Faisal Specialist Hospital and Research Centre, Riyadh; Departments of Women's Health (SEA) and Pediatrics (SHM) and the PGD Laboratory (AMH), Saad Specialist Hospital, Al-Khobar, Saudi Arabia. Received June 20, 2012. Final Revisions Submitted August 24, 2012. Correspondence to: Khaled K. Abu-Amero, Department of Ophthalmology, College of Medicine, King Saud University, Riyadh, Saudi Arabia. Email: abuamero@gmail.com.
} 


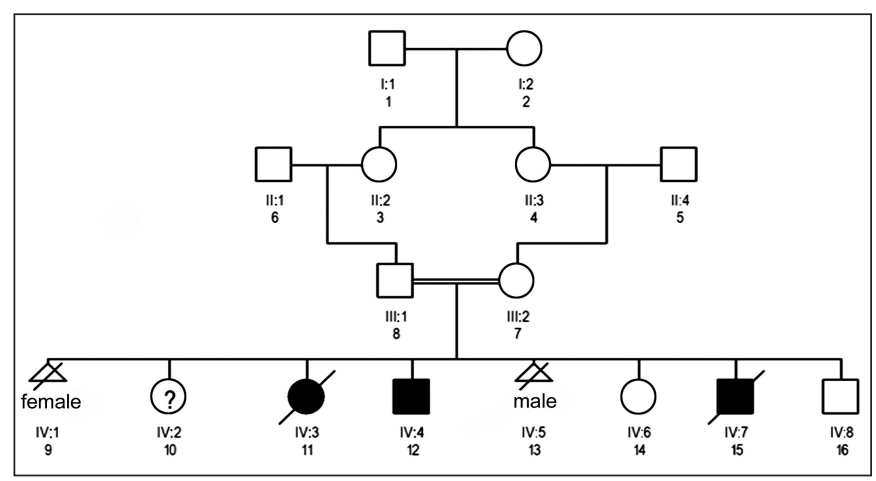

Figure 2: Family pedigree. Pedigree of consanguineous Sudanese family followed for eight years who had three children with clinically and biochemically defined ISOD.

with effacement of cerebral sulci and compression of the bodies of the lateral ventricles (Figure 3A) with later development of cystic changes in the cerebral hemispheres, white matter loss, enlargement of sulci, and thalamic calcification when imaged at age of three years and eight months (Figure 3B). Urinary Ssulphocysteine was elevated (144 $\mu \mathrm{mol} / \mathrm{mmol}$ of creatinine) while urinary xanthine and hypoxanthine were normal, diagnostic of ISOD. At age ten months he had microcephaly associated with evolving facial dysmorphism (Figure 4A), gross motor delay, and diffuse hypotonia with brisk deep tendon reflexes. He remained vegetative and oxygen-dependent at age eight years.

The fifth pregnancy was a genetically normal male fetus (Individual 13) that aborted at three months gestation after diagnostic chorionic villus sampling performed at ten weeks. The sixth pregnancy yielded a normal girl (Individual 14), while the seventh child was a boy with biochemically proven ISOD who began to seize on the second day of life and died on day 15 (Individual 15). The eighth pregnancy was achieved after PGD and yielded a normal male child (Individual 16; Figure 4B).

\section{Materials ANd Methods}

Medical records and neuroimaging were reviewed for the two deceased children and the one living child with ISOD. After giving informed consent, the parents chose to undergo mutation screening in hope of obtaining PGD.

\section{Mutation Detection}

Genomic DNA was extracted from blood samples of two patients with ISOD (Individuals 11 and 12), their parents (Individuals 7 and 8), and control samples, and polymerase chain reaction (PCR) amplification of the three exons of the SUOX coding region and exon-intron boundaries was performed utilizing primers described previously ${ }^{2}$. All the sequenced fragments were analyzed utilizing SeqScape software v2.6 (Applied Biosystems).

\section{Blastomere Diagnosis}

Controlled ovarian stimulation, fertilization, embryo culture, and biopsy were accomplished as previously described ${ }^{3}$. Seven

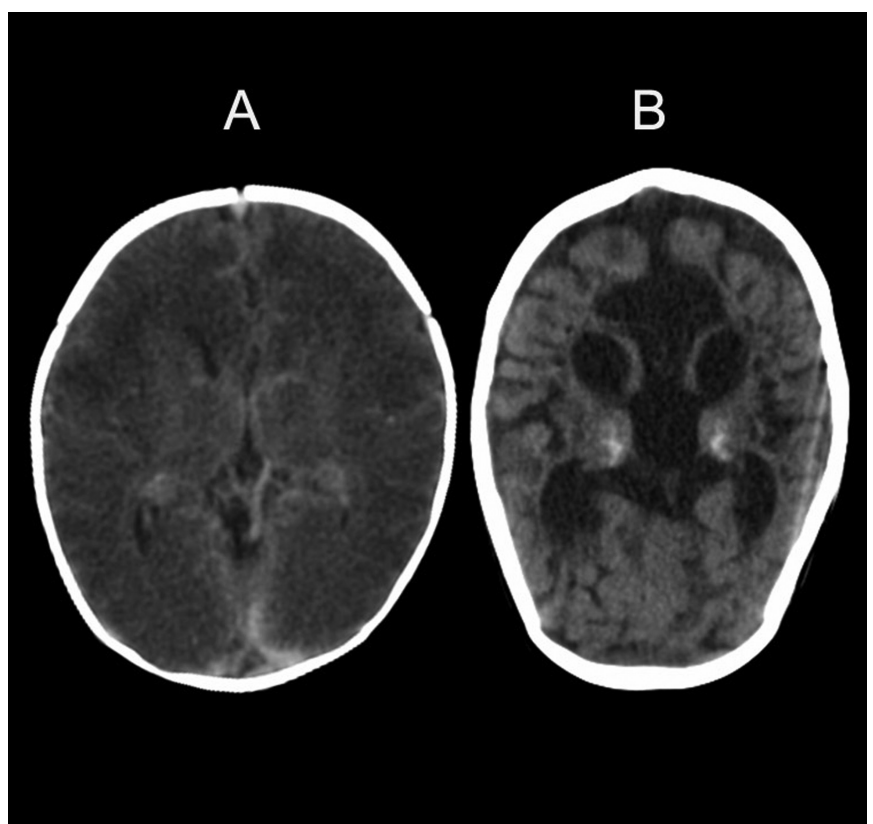

Figure 3: Neuroimaging. A) Axial CT of the brain at the age of four days showing diffuse brain swelling causing effacement of cerebral sulci and compression of the lateral ventricles. White matter, basal ganglia, and thalami have remarkable low attenuation. B) Follow up at the age of three years and eight months revealed progressive white matter loss with associated enlargement of the lateral ventricles, cisterns, sulci, and fissures. Cysts are seen in the white matter and basal ganglia. Faint calcification is evident in both thalami.

oocytes collected were injected with the father's sperm using the intra-cytoplasmic sperm injection technique to eliminate contamination by sperm when performing subsequent embryo biopsy.

The zona pellucida was pierced using the Saturn Laser System (Research Instrument, Cornwall, UK), and two blastomeres were carefully aspirated from five embryos. Whole genome amplification using Multiple Displacement Amplification (MDA; GE Healthcare, Waukesha, WI, USA) was carried out on each blastomere lystae. The resultant MDA product was diluted to a concentration of $10 \mathrm{ng} / \mu \mathrm{l}$ and each embryonic DNA studied was amplified by PCR using primers covering the mutation. PCR amplifications were sequenced and analyzed on 3130xl genetic analyzer.

\section{RESULTS}

\section{Mutation results}

After sequencing the SUOX gene in both parents (Individuals 7 and 8) and two affected children (Individuals 11 and 12), a two base successive deletion c.1232-1233 delTG was detected that was homozygous in the affected children, heterozygous in both parents, and not detected in 100 chromosomes from individuals of matching ethnicity. This deletion will lead to frame shifting of the original codons ACT GTA GAT TTT GAC (which normally encode amino acids 410-414: TVDFD) to generate the new codons ACT AGA TTT TGA (which will encode TRF*) in the mutated protein, resulting in truncation of the molybdopterin 


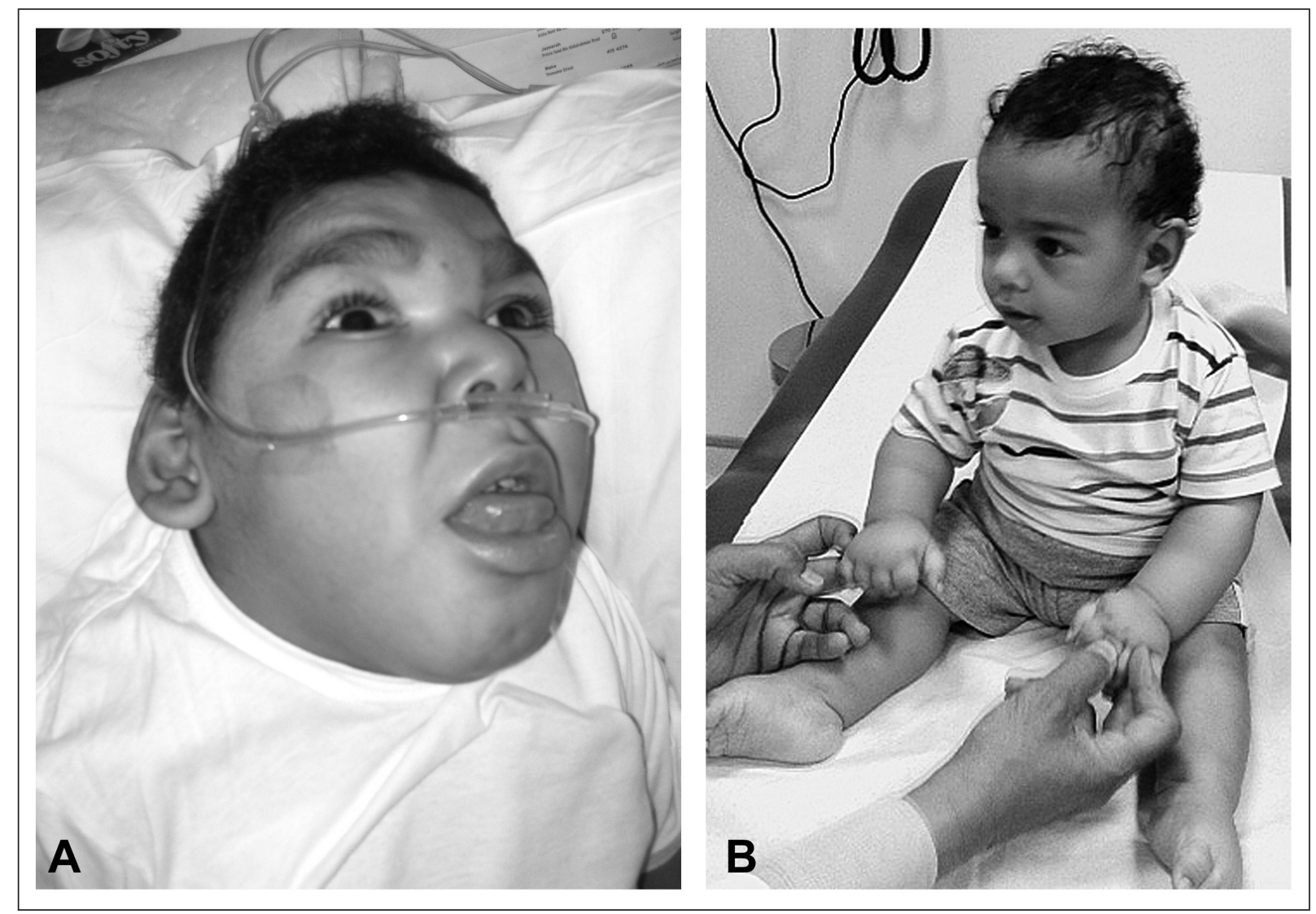

Figure 4: 4 ISOD phenotype. A) Photograph illustrating the dysmorphic features of Individual 12 at the age of 7 years, including narrow bifrontal diameter, deep-set eyes, coarse features, large ears, and large tongue. B) Photograph of Individual 16, a normal 9month-old boy born following successful PGD.

binding domain of sulfite oxidase protein. This novel mutation has not been reported previously and is not found in the human genome mutation database (HGMD; http://www.hgmd.org/). All four affected codons (410-414) are highly conserved among other species (http://genome.ucsc.edu).

\section{Preimplantation Genetic Diagnosis}

The parents underwent PGD with in vitro fertilization, and the resultant pregnancy yielded a phenotypically and genetically normal male child (Individual 16).

\section{Discussion}

We describe a consanguineous family who had three children with clinical and biochemical evidence of ISOD, two of whom have died and the third of whom is vegetative. They also had an anencephalic baby and one child born prematurely, both of whom may have had ISOD but died without diagnosis. Two children with ISOD were homozygous for a novel two nucleotide deletion in the SUOX gene, and the parents were both heterozygous for this mutation, confirming the genetic diagnosis of ISOD in this family. Phenotypic variability, from early death to prolonged survival in a vegetative state, is common in ISOD. Sequencing of the SUOX gene revealed a novel mutation, and subsequent PGD was able to isolate embryos without homozygous SUOX mutations for in vitro fertilization, resulting in a normal child.

A patient with ISOD typically presents as a normal-appearing infant after an uneventful delivery who develops intractable seizures and failure to thrive within the first days of $l^{1} \mathrm{fe}^{1}$. The child then becomes microcephalic ${ }^{2}$ over a period of months with evolving dysmorphic features (Figure 3A) and severe developmental delay due to progressive brain injury. Most children die within the first two years of life, while survivors have severe developmental delay. A family's first child with ISOD may be misdiagnosed because the clinical and neuroimaging presentation is similar to that of perinatal hypoxicischemic brain damage, and the diagnosis is commonly considered only after a second child is similarly affected or the first child develops dislocated lenses during the first months or years of life. Biochemical confirmation of ISOD is based on the presence of elevated urinary S-sulfocysteine levels with normal levels of xanthine and hypoxanthine ${ }^{4}$.

Neuroimaging and neuropathology are compatible with this neurologic presentation, revealing dramatic, progressive damage to cerebral hemispheric white and grey matter that appears as diffuse brain edema shortly after birth and usually progresses to cystic replacement of much of the cerebral hemispheres. The 
cerebellum and corpus callosum are typically small on initial neuroimaging, implying in utero hypoplasia preceding the fulminant post-partum injury to other parts of the brain.

ISOD currently has limited treatment options. Seizure control is typically incomplete despite multiple anticonvulsants. An attempt to reduce intake of sulfur-containing amino acids with a low protein diet may have helped two mildly affected individuals ${ }^{5}$ but not other patients. Most importantly, no dietary adjustment can affect hypoplasia of the cerebellum, corpus callosum, and cerebral cortex that are present at birth .

This family's tragic story illustrates the clinical severity of this neurogenetic disorder and the importance of genetic diagnosis in permitting genetic counseling even when the syndrome in question is not currently treatable. This family finally had a normal child after PGD, illustrating the unique value the approach to this and other genetic problems that cause significant brain and other developmental abnormalities in utero or in very early infancy.

\section{REFERENCES}

1. Sass JO, Gunduz A, Araujo Rodrigues Funayama C, et al. Functional deficiencies of sulfite oxidase: Differential diagnoses in neonates presenting with intractable seizures and cystic encephalomalacia. Brain Dev. 2010 Aug;32(7):544-9.

2. Seidahmed MZ, Alyamani EA, Rashed MS, et al. Total truncation of the molybdopterin/dimerization domains of SUOX protein in an Arab family with isolated sulfite oxidase deficiency. Am J Med Genet A. 2005 Jul 15;136(2):205-9.

3. Hellani A, Sammour A, Johansson L, El-Sheikh A. Delivery of a normal baby after preimplantation genetic diagnosis for nonketotic hyperglycinaemia. Reprod Biomed Online. 2008 Jun;16 (6):893-7.

4. Rashed MS, Saadallah AA, Rahbeeni Z, et al. Determination of urinary S-sulphocysteine, xanthine and hypoxanthine by liquid chromatography-electrospray tandem mass spectrometry. Biomed Chromatogr. 2005 Apr;19(3):223-30.

5. Touati G, Rusthoven E, Depondt E, et al. Dietary therapy in two patients with a mild form of sulphite oxidase deficiency. Evidence for clinical and biological improvement. J Inherit Metab Dis. 2000 Feb;23(1):45-53. 